\title{
Neuron specific enolase is a potential target for regulating neuronal cell survival and death: implications in neurodegeneration and regeneration
}

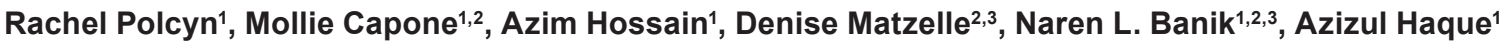 \\ ${ }^{1}$ Department of Microbiology and Immunology, Hollings Cancer Center, Medical University of South Carolina, Charleston, SC 29425, USA. \\ ${ }^{2}$ Department of Neurosurgery, Medical University of South Carolina, Charleston, SC 29425, USA. \\ ${ }^{3}$ Ralph H. Johnson Veterans Administration Medical Center, Charleston, SC 29401, USA.
}

Correspondence to: Dr. Azizul Haque, Department of Microbiology and Immunology, Hollings Cancer Center, Medical University of South Carolina, 173 Ashley Avenue, BSB-201, Charleston, SC 29425, USA. E-mail: haque@musc.edu

How to cite this article: Polcyn R, Capone M, Hossain A, Matzelle D, Banik NL, Haque A. Neuron specific enolase is a potential target for regulating neuronal cell survival and death: implications in neurodegeneration and regeneration. Neuroimmunol Neuroinflammation $2017 ; 4: 254-7$.

Article history: Received: 17 Nov 2017 Accepted: 17 Nov 2017

Enolase is a multifunctional enzyme primarily involved in catalyzing the conversion of 2-phosphoglycerate to phosphoenolpyruvate during glycolysis and the reverse reaction during gluconeogenesis ${ }^{[1-4]}$. Though typically expressed in the cytosol, enolase has been shown to migrate to the cell surface upon inflammatory signal ${ }^{[3]}$. It then enhances antigen presentation for the invasion of host cells via plasminogen binding and subsequent plasmin activation, leading to degradation of the extracellular matrix. Cell surface expression of enolase, possibly due to an association with the urokinase-type plasminogen activator (UPA)/ UPA receptor complex, additionally induces the production of reactive oxygen species, nitric oxide, and pro-inflammatory cytokines [tumor necrotic factor (TNF)- $\alpha$, interleukin (IL)-1 $\beta$, interferon- $\gamma$, and transforming growth factor- $\beta$ ] and chemokines [monocyte chemotactic protein 1 and macrophage inflammatory protein (MIP)-1 $\alpha$ ] to augment neurodegenerative response ${ }^{[3,5]}$. Lysosomal proteases,
Published: 6 Dec 2017

especially cathepsins (e.g. Cathepsin X or Cat X), are instrumental in processing several neuronal proteins that generate either harmful or neuroprotective functions. Cathepsins and a neutral protease, calpain, also have regulatory functions in antigen processing and presentation, thereby inducing immune responses that can have either detrimental or beneficial effects on neuronal cells. This editorial discusses the relationships between neuron specific enolase (NSE) and $C$ at $X$ activity in neuronal cells with a special focus on their implications for neurodegeneration and neuroprotection.

Distinct dimeric isoforms of enolase are composed of non-covalently linked $\alpha, \beta$, or $y$ subunits and are tissue-specific ${ }^{[3]}$. During development, injury, or disease, $\alpha$-enolase (enolase 1, non-neuronal enolase, ENO1), which is primarily found in adult tissue, can be converted to $\mathrm{y}$-enolase (enolase 2, NSE) in neurons and neuroendocrine cells. Similarly, a-enolase is License (https://creativecommons.org/licenses/by/4.0/), which permits unrestricted use, distribution, and reproduction in any medium, as long as the original author is credited and the new creations are licensed under the identical terms.

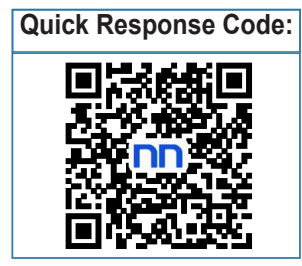


converted into $\beta$-enolase (enolase 3 , muscle specific enolase, ENO3) in muscle tissue. NSE exists as both ay and $y y$ isoforms in neurons, $y y$ in microglia and oligodendrocytes, and $\alpha y$ in astrocytes ${ }^{[3,4]}$. Notably, enolase levels have been shown to be significantly increased in astrocytes and microglia following spinal cord injury ( $\mathrm{SCl}$ ), an observation indicating a possible role for NSE in different pathologies associated with the neuroinflammatory, apoptotic, and neuroprotective activity of these interacting glial cells ${ }^{[6,7]}$. Due to its specific location in neurons and neuroendocrine cells and its upregulated secretion following axonal damage, NSE has been implicated as a biomarker of functional damage to neurons in $\mathrm{SCl}$ and several other pathophysiological conditions: traumatic brain injury, stroke, ischemia-reperfusion injury, cardiac arrest, neuroblastoma, small-cell lung cancer, and Alzheimer's disease $(A D)^{[2-5]}$.

$\mathrm{SCl}$ is a debilitating neurological disorder that occurs in two main phases: primary and secondary injury ${ }^{[3,8]}$. The initial primary injury, resulting from laceration, contusion, compression, and/or contraction of the neural tissue, is the immediate, irreversible damage to the spinal cord and associated axons, cells, and blood vessels. However, a diverse array of secondary injury mechanisms, including hypoxia, ischemia, excitotoxicity, inflammation, and apoptosis, expands the injury site and impairs pro-survival activity following primary injury ${ }^{[9]}$. These secondary processes are reversible and as such have been a principle target of $\mathrm{SCl}$ treatment research ${ }^{[8]}$. Substantial reduction in blood supply from primary injury triggers ischemia, oxidative stress, and microglial activation that drive the release of pro-inflammatory cytokines and chemokines at the injury site. Under the hypoxicischemic conditions immediately following $\mathrm{SCl}$, an influx of $\mathrm{Ca}^{2+}$ results in activation of calpain, caspase, and phospholipases ${ }^{[10,11]}$. Calpain then degrades cytoskeletal proteins and leads to apoptosis. Additional neuronal death can be attributed to glutamate excitotoxicity following the injury ${ }^{[9]}$. Though these effects are primarily neurodegenerative, macrophages may function as pro- or anti-inflammatory agents in $\mathrm{SCl}$, depending on the M1/M2 macrophage cell ratio in the injured microenvironment.

Our group has shown that $\mathrm{SCI}$ treatment in a male Sprague-Dawley rat model using ENOblock, a small molecule inhibitor of enolase, corresponded to a reduction of NSE expression in SCI tissues and a significant decrease in serum NSE, matrix metallopeptidase (MMP)-9 protein expression in injured tissue, serum pro-inflammatory cytokines
(TNF- $\alpha, \mathrm{IL}-1 \beta$, and IL-6) and chemokines (MIP$1 \alpha$ and $(P-10)$, and glial activation ${ }^{[2]}$. Elevated MMP-9 expression can promote the activation of microglia and astrocytes, leading to the release of inflammatory cytokines and chemokines that promote cell death. Expression of these pro-inflammatory cytokines (TNF- $\alpha, \mathrm{IL}-1 \beta$, and IL-6) is known to induce hyperalgesia, allodynia, and apoptosis of neuronal and glial cells in association with the secondary damages of $\mathrm{SCl}^{[2,12-15]}$. Additionally, MIP-1 $\alpha$ has been shown to mediate microglia accumulation and neuroinflammation in brain injury ${ }^{[16]}$. IP-10, expressed by astrocytes in response to $\mathrm{N}$-methyl-D-aspartatedependent excitotoxicity, activates the mitogenactivated protein kinase (MAPK)/extracellular signalregulated kinase (ERK) pathway in mouse cortical neurons, indicating a role for this chemokine in mediating cell proliferation and apoptosis under neurodegenerative conditions ${ }^{[17,18]}$. The reduction of MMP-9 and the aforementioned pro-inflammatory cytokines and chemokines by ENOblock indicates the potential for this treatment in attenuating neural damages associated with inflammatory response during secondary injury mechanisms of $\mathrm{SCl}$.

While previous research on the role of NSE in secondary injury mechanisms of $\mathrm{SCl}$ has focused on neurodegenerative effects, namely the association between the overexpression of NSE and an inflammatory cascade leading to neuronal cell death, future studies should additionally investigate the role of NSE in pro-survival and regeneration activity via cellular signaling pathways in acute and chronic SCI. NSE has been shown to exhibit neurotrophic activity in controlling neuronal survival, differentiation, and neurite regeneration of human neuroblastoma SH-SY5Y cells via activation of the phosphoinositide 3-kinase (PI3K)/Akt and MAPK/ ERK signaling pathways, which regulate cytoskeleton reorganization and transcriptional factor activation in promotion of cell survival and neurite outgrowth ${ }^{[1]}$. A neuroprotective effect of NSE expression and activity was also observed in a mouse model of $A D$ against amyloid- $\beta$-related neurodegeneration ${ }^{[5]}$. Additionally, the neuritogenic activity of NSE is associated with RhoA kinase inactivation within the PI3K/ Akt pathway and affects neurite outgrowth through rapid actin polymerization ${ }^{[1]}$. NSE likely exhibits similar neuroprotective activity for cell survival, differentiation, and migration following $\mathrm{SCl}$ and other neurodegenerative conditions via the PI3K/Akt and MAPK/ERK pathways. Further research evaluating the interaction between NSE and these pathways in $\mathrm{SCl}$ secondary injury mechanisms for more clarification would be interesting. 
Investigation into the neurotrophic activity of NSE in a mouse model of the neurodegenerative condition $A D$ has shown that NSE can be regulated by Cat $X$, a lysosomal cysteine protease that cleaves the $C$-terminal end of the NSE enzyme under acidic conditions $^{[5]}$. The C-terminal peptide of NSE, which is not involved in plasminogen binding (due to the absence of lysine) or glycolytic function, contains a PDZ-binding domain for the scaffold protein $y-1$ syntrophin that enables NSE to relocate to the plasma membrane via actin filament, as evidenced by their colocalization ${ }^{[4]}$. This $\mathrm{C}$-terminal peptide has been shown to have a pro-survival effect on PC12 cells ${ }^{[19]}$. The cleavage of NSE at this site by Cat X severely affects its ability to function in neuronal cell differentiation for pro-survival activity or cell death. Because of the known involvement of PI3K/Akt and MAPK/ERK signaling pathways in the activation of cathepsin $B$, a similar cysteine protease, in glioma, Cat $X$ activity is likely associated with these same pathways ${ }^{[20]}$.

An additional cysteine protease, calpain, is involved in the neuroinflammatory response to $\mathrm{SCl}^{[21]}$. Calpain is found in the cytosol and is active under neutral $\mathrm{pH}$ conditions upon $\mathrm{Ca}^{2+}$ activation. The role of calpain in apoptosis has been clearly demonstrated, and its activation in $\mathrm{SCl}$ conditions has been shown to lead to cytoskeletal and myelin protein cleavage. Calpeptin, a calpain inhibitor, can exhibit neuroprotective effects against excitotoxic apoptosis, reducing neuronal cell death ${ }^{[22]}$. While inhibition of enolase by ENOblock alters cellular growth, cytokines/chemokines, and inflammatory markers ${ }^{[2,23]}$, it is unknown if ENOblock acts on Cat $X$ and regulates its function. Our group has found increased calpain activity and cell-specific overexpression in astrocytes, microglia, macrophages and $T$ cells in inflammatory demyelinating diseases ${ }^{[24-26]}$. However, the effects of calpain inhibition (calpeptin) on NSE and Cat X functions remain to be investigated. Calpeptin, which is a cysteine protease inhibitor, could possibly target Cat $X$, leading to inhibition of NSE-mediated inflammatory events and promotion of neuronal cell survival. Since NSE is a substrate of Cat $X$, evaluating both ENOblock and calpeptin as potential mediators of NSE expression and activity in neuronal cells following $\mathrm{SCl}$ and other neurological disorders.

At certain levels, NSE can support regeneration of neuronal cells ${ }^{[1,5]}$. NSE-mediated activation of the PI3K/Akt and MAPK/ERK pathways likely supports cell survival and regeneration. On the other hand, these pathways also likely activate Cat $X$, an enzyme that cleaves NSE. Cat $X$ activity would likely result in a reduction of NSE-mediated activation of PI3K/Akt and
MAPK/ERK pathways and result in cell death. It would be interesting to investigate the role of ENOblock in regulating PI3K/Akt and MAPK/ERK pathways and Cat $X$ activity. Future studies to elucidate the role of PI3K/Akt and MAPK/ERK pathways in defining cell fate are warranted. Mediation of the Cat $X$ activity in association with these pathways could result in partial as opposed to total degradation of NSE, thus reducing NSE and Cat $X$ mediated cell death and providing a promising future therapeutic target for reversal of secondary injury mechanisms in acute and chronic $\mathrm{SCl}$ as well as other neurological conditions.

In conclusion, several future avenues for research on the mechanisms of NSE expression and activity in neurons and glia and the process of neurodegeneration and regeneration following neurological impairment have been discussed. NSE, once migrated to the plasma membrane, takes part in cellular activation, production of inflammatory cytokines and chemokines, and induction of neuronal cell death (neurodegeneration) ${ }^{[1,3]}$. The regulated expression of NSE may promote neuronal survival (neuroprotection or regeneration) via cell survival pathways. Previous research has focused on the harmful effects of NSE overexpression following neuronal damage. However, future studies should address the conditions leading to preferential differentiation into pro-survival activity or neuronal cell death and specific methods for regulating NSE and Cat $X$ activity to mediate the secondary damages associated with $\mathrm{SCl}$. The role of Cat $X$ in secondary injury remains unknown, as does the influence of $\mathrm{SCl}$ on Cat $\mathrm{X}$ expression and activity. Additionally, the direct and indirect targets of ENOblock treatment have yet to be determined. Calpeptin, which acts on calpain to reduce neuronal cell death, may similarly act on Cat $X$ to regulate NSE activity. The effects of these inhibitors on neurodegeneration and/or neuroprotection and their potential interaction with the PI3K/Akt and MAPK/ERK pathways remain to be determined. This editorial has highlighted several potential intermediary effectors associated with neurodegeneration and neuroprotection in $\mathrm{SCl}$ and other neuropathological conditions. Significant research is needed to further evaluate these possible mechanisms and their potential for translation into future preclinical and clinical treatments.

\section{DECLARATIONS}

\section{Authors' contributions}

Overall design and completion of the manuscript: A. Haque

Performed original research on enolase and $\mathrm{SCl}$, and analyzed data published in Neurochemical 
Research-2017: M. Capone, A. Hossain, D. Matzelle

Drafted the manuscript: R. Polcyn, A. Haque

Edited the manuscript: D. Matzelle, N.L. Banik

Reviewed and approved the final version of the manuscript: All authors

\section{Financial support and sponsorship}

This work was supported by grants from the National Institutes of Health (R01 CA129560) and the South Carolina Spinal Cord Injury Research Fund (SCIRF \#2016 I-03), MUSC Center for Global Health Award, and the HCC-NCl Incentive Award to A. Haque, and Ralph $\mathrm{H}$. Johnson Veterans Administration Medical Center, Charleston, SC (1101BX002349-01) to N.L. Banik.

\section{Conflicts of interest \\ There are no conflicts of interest.}

\section{Patient consent \\ Not applicable.}

\section{Ethics approval \\ Not applicable.}

\section{REFERENCES}

1. Hafner A, Obermajer N, Kos J. gamma-Enolase C-terminal peptide promotes cell survival and neurite outgrowth by activation of the PI3K/ Akt and MAPK/ERK signalling pathways. Biochem J 2012;443:43950 .

2. Haque A, Capone M, Matzelle D, Cox A, Banik NL. Targeting enolase in reducing secondary damage in acute spinal cord injury in rats. Neurochem Res 2017;42:2777-87.

3. Haque A, Ray SK, Cox A, Banik NL. Neuron specific enolase: a promising therapeutic target in acute spinal cord injury. Metab Brain Dis 2016;31:487-95.

4. Vizin T, Kos J. Gamma-enolase: a well-known tumour marker, with a less-known role in cancer. Radiol Oncol 2015;49:217-26.

5. Hafner A, Glavan G, Obermajer N, Zivin M, Schliebs R, Kos J. Neuroprotective role of gamma-enolase in microglia in a mouse model of Alzheimer's disease is regulated by cathepsin X. Aging Cell 2013;12:604-14.

6. Li M, Wen H, Yan Z, Ding T, Long L, Qin H, Wang H, Zhang F. Temporal-spatial expression of ENOLASE after acute spinal cord injury in adult rats. Neurosci Res 2014;79:76-82.

7. Shinozaki Y, Shibata K, Yoshida K, Shigetomi E, Gachet C, Ikenaka K, Tanaka KF, Koizumi S. Transformation of astrocytes to a neuroprotective phenotype by microglia via P2Y1 receptor downregulation. Cell Rep 2017;19:1151-64.

8. Varma AK, Das A, Wallace Gt, Barry J, Vertegel AA, Ray SK, Banik NL. Spinal cord injury: a review of current therapy, future treatments, and basic science frontiers. Neurochem Res 2013;38:895-905.
9. Cox A, Varma A, Banik N. Recent advances in the pharmacologic treatment of spinal cord injury. Metab Brain Dis 2015;30:473-82.

10. Chakrabarti M, Haque A, Banik NL, Nagarkatti P, Nagarkatti M, Ray SK. Estrogen receptor agonists for attenuation of neuroinflammation and neurodegeneration. Brain Res Bull 2014;109:22-31.

11. Momeni HR. Role of calpain in apoptosis. Cell J 2011;13:65-72.

12. Zhang JM, An J. Cytokines, inflammation, and pain. Int Anesthesiol Clin 2007;45:27-37.

13. Sun S, Chen D, Lin F, Chen M, Yu H, Hou L, Li C. Role of interleukin-4, the chemokine CCL3 and its receptor CCR5 in neuropathic pain. Mol Immunol 2016;77:184-92.

14. Sun Y, Yang M, Tang H, Ma Z, Liang Y, Li Z. The over-production of TNF-alpha via Toll-like receptor 4 in spinal dorsal horn contributes to the chronic postsurgical pain in rat. $J$ Anesth 2015;29:734-40.

15. Teixeira JM, de Oliveira-Fusaro MC, Parada CA, Tambeli $\mathrm{CH}$ Peripheral $\mathrm{P} 2 \mathrm{X} 7$ receptor-induced mechanical hyperalgesia is mediated by bradykinin. Neuroscience 2014;277:163-73.

16. Zhu X, Wei D, Chen O, Zhang Z, Xue J, Huang S, Zhu W, Wang Y Upregulation of CCL3/MIP-1alpha regulated by MAPKs and NFkappaB mediates microglial inflammatory response in LPS-induced brain injury. Acta Neurobiol Exp (Wars) 2016;76:304-17.

17. Liu C, Cui G, Zhu M, Kang X, Guo H. Neuroinflammation in Alzheimer's disease: chemokines produced by astrocytes and chemokine receptors. Int J Clin Exp Pathol 2014;7:8342-55.

18. van Weering HR, Boddeke HW, Vinet J, Brouwer N, de Haas AH, van Rooijen N, Thomsen AR, Biber KP. CXCL10/CXCR3 signaling in glia cells differentially affects NMDA-induced cell death in CA and DG neurons of the mouse hippocampus. Hippocampus 2011;21:220-32.

19. Obermajer N, Doljak B, Jamnik P, Fonovic UP, Kos J. Cathepsin X cleaves the C-terminal dipeptide of alpha- and gamma-enolase and impairs survival and neuritogenesis of neuronal cells. Int $J$ Biochem Cell Biol 2009;41:1685-96

20. Malla R, Gopinath S, Alapati K, Gondi CS, Gujrati M, Dinh DH, Mohanam S, Rao JS. Downregulation of uPAR and cathepsin B induces apoptosis via regulation of $\mathrm{Bcl}-2$ and $\mathrm{Bax}$ and inhibition of the PI3K/Akt pathway in gliomas. PLoS One 2010;5:e13731.

21. Yu CG, Li Y, Raza K, Yu XX, Ghoshal S, Geddes JW. Calpain 1 knockdown improves tissue sparing and functional outcomes after spinal cord injury in rats. J Neurotrauma 2013;30:427-33.

22. D'Orsi B, Bonner H, Tuffy LP, Dussmann H, Woods I, Courtney MJ, Ward MW, Prehn JH. Calpains are downstream effectors of baxdependent excitotoxic apoptosis. J Neurosci 2012;32:1847-58.

23. Cho H, Um J, Lee JH, Kim WH, Kang WS, Kim SH, Ha HH, Kim YC Ahn YK, Jung DW, Williams DR. ENOblock, a unique small molecule inhibitor of the non-glycolytic functions of enolase, alleviates the symptoms of type 2 diabetes. Sci Rep 2017;7:44186.

24. Trager N, Smith A, Wallace Iv G, Azuma M, Inoue J, Beeson C, Haque A, Banik NL. Effects of a novel orally administered calpain inhibitor SNJ-1945 on immunomodulation and neurodegeneration in a murine model of multiple sclerosis. J Neurochem 2014;130:268-79.

25. Smith AW, Rohrer B, Wheless L, Samantaray S, Ray SK, Inoue J, Azuma M, Banik NL. Calpain inhibition reduces structural and functional impairment of retinal ganglion cells in experimental optic neuritis. J Neurochem 2016;139:270-84.

26. Podbielska M, Das A, Smith AW, Chauhan A, Ray SK, Inoue J, Azuma M, Nozaki K, Hogan EL, Banik NL. Neuron-microglia interaction induced bi-directional cytotoxicity associated with calpain activation. $J$ Neurochem 2016;139:440-55. 